\title{
Masses of the Elbow and Forearm
}

UNIT A27.2

MRI is valuable for mass localization and evaluation because of its multiplanar capabilities and soft-tissue detail. While in many instances the imaging findings may not be specific, MRI can be very sensitive. The technique used plays a vital role in the pathway to the differential diagnosis and staging of a mass lesion by defining the precise anatomic involvement, relationships, and intrinsic characteristics.

\section{IMAGING OF THE ELBOW}

As in routine imaging of the elbow, a dedicated extremity coil is preferred. The sequences should include $T_{1}$ - and $T_{2}$-weighted sequences for tissue characterization. Fatsuppression images should also be included to increase sensitivity as well as for possible fat tissue characterization. Images should be obtained in at least two and preferably all three planes for anatomic demonstration and involvement. The transverse plane should always be included. Additional long axis planes would include sagittal for antero-posterior masses and coronal for medio-lateral masses; if possible, both are generally helpful. $T_{1}$-weighted images are vital in tumor evaluation because of the anatomic soft tissue and cortical detail and for demonstration of fat or hemorrhage components. It is this anatomic and tissue definition that may aid the diagnosis or in determining the diagnostic category, in addition to providing a map for possible surgical management. A fat-saturated $T_{2}$ weighted or STIR (short-tau inversion recovery) sequence is also recommended. STIR sequences should be substituted for the fat-saturated sequences to achieve more homogeneous fat suppression when field uniformity may be poor. With lower-field scanners, where fat saturation sequences may not be available, STIR sequences generally still provide good fat suppression. The fat-suppressed longer $T_{\mathrm{E}}\left(T_{2}\right.$-weighted) and STIR sequences are highly sensitive to marrow edema. A $T_{2}$-weighted sequence without fat suppression in one of the three planes is helpful to assist in demonstrating true high-signal fluid in a cystic or necrotic lesion (Kneeland, 1997; Steinbach et al., 1997a).

Table A27.2.1 lists the hardware necessary to perform the procedure, along with appropriate parameters. The available gradient strength will depend on the scanner, and the echo times given in other tables below may be varied accordingly (the smaller the gradient strength, the longer the echo time for a particular scan).

In summary, our standard protocol consists of coronal and sagittal $T_{1}$-weighted and fat-saturated $T_{2}$-weighted (or STIR) images, transverse proton density- and $T_{2}$-weighted images, as detailed below. Total exam time is $\sim 25 \mathrm{~min}$.

NOTE: Be sure that technologists and nurses have immediate access to any emergency equipment that may be relevant to a given study, or that may be needed for a particular patient, such as crash carts or oxygen.

Table A27.2.1 Equipment Parameters for MRI of the Elbow

\begin{tabular}{ll}
\hline Coil type & Phased array extremity coil \\
Gradient coil strength & $25 \mathrm{mT} / \mathrm{m}$ (or whatever the system permits) \\
Cardiac gating & No \\
Peripheral gating & No \\
Respiratory gating & No \\
Oxygen & No \\
Motion cushions & Useful \\
Use of contrast agent & No (but used in Alternate Protocol) \\
\hline
\end{tabular}

BASIC

PROTOCOL 


\section{Set up patient and equipment}

1. Interview (screen) the patient to ensure that there are no contraindications for the MRI exam such as cardiac pacemakers, cerebral aneurysm clips, biostimulators, and metallic bodies within the orbit, or other electronic devices sensitive to magnetic fields. Question the patient regarding medical conditions that may require emergency care or equipment, in addition to conditions that would inhibit the patient from being able to complete the exam.

Generally, standard screening forms (see APPENDIX 1) are used for all patients scanned in a magnetic resonance system.

The presence of any ferromagnetic metals may be a health hazard to the patient when he or she is inside the magnet, and will also affect the imaging. If in doubt as to the exact composition of the items, it is best to exclude patients with any metal implants; see Shellock (2001) for discussion of what implants may be safely scanned using magnetic resonance. Orthopedic hardware is not a contraindication. However, ferromagnetic and even nonferromagnetic material in this hardware will produce artifacts that may degrade the exam. Adjustments to the technique may be made as discussed below.

Patients may be accompanied into the magnet room by a friend or family member, who can sit in the room during the scan and comfort the patient as needed. This companion must be screened as well to ensure the absence of loose metal objects on the body or clothing.

2. If the procedure is a research protocol, have the patient sign any necessary consent forms.

3. Have the patient remove all jewelry and clothing items or accessories that may contain metal and change into a gown to eliminate the presence of any metal that might be found in clothing. Remove pants due to the positioning of the arm adjacent to the hip.

4. Have the patient wash off any mascara and other makeup to avoid local tissue heating and image artifacts.

5. Inform the patient about what will occur during the procedure, what he or she will experience while in the magnet, and how to behave, including the following:

a. If earplugs or headphones are used to protect the ears from the loud sounds produced by the gradients, the patient will be asked to wear these, but will be able to communicate with you at any time during the imaging.

b. Establish a method of communication with the patient using either an intercom or signaling device.

c. In order to obtain good results, the patient should not talk, and should avoid or minimize other movement, during each scan-i.e., as long as the banging sounds continue. Between scans, talking is allowed in most cases, but should be avoided when comparative positional studies are being performed; the patient will be informed when this is the case.

d. Nevertheless, the patient may call out at any time if he or she feels it necessary.

6. Tape a surface marker (a cod liver oil or vitamin E pill) over the symptomatic site or palpable abnormality. Do not compress a superficial mass.

The cod liver oil pill shows up better on STIR sequences.

7. Have the patient lie supine with the forearm in supination at his or her side. Place the elbow in full extension into the RF coil with the antecubital fossa in the center of the coil; the patient's arm (not necessarily the body) should be straight and parallel to the scanning table; the arm will be parallel to the main field for a cylindrical magnet 
Table A27.2.2 Primary Clinical Imaging Parameters for Sequence 1 (Localizer)

\begin{tabular}{|c|c|}
\hline Patient position & Supine \\
\hline Scan type & 2-D gradient echo \\
\hline Imaging plane (orientation) & $\begin{array}{l}\text { Three planes (if unavailable use } \\
\text { transverse plane) }\end{array}$ \\
\hline Central slice or volume center & Center of mass \\
\hline Echo time $\left(T_{\mathrm{E}}\right)$ & $5 \mathrm{msec}$ \\
\hline Repeat time $\left(T_{\mathrm{R}}\right)$ & $15 \mathrm{msec}$ \\
\hline Flip angle (FA) & $40^{\circ}$ \\
\hline Fields of view $\left(\mathrm{FOV}_{\mathrm{x}}, \mathrm{FOV}_{\mathrm{y}}\right)$ & $\begin{array}{l}200 \mathrm{~mm}, 200 \mathrm{~mm} \text { (varies with the } \\
\text { mass) }\end{array}$ \\
\hline Resolution $(\Delta x, \Delta y)$ & $0.78 \mathrm{~mm}, 1.56 \mathrm{~mm}$ \\
\hline Number of data points collected $\left(N_{\mathrm{x}}, N_{\mathrm{y}}\right)$ & 256,128 \\
\hline Slice thickness $(\Delta z)$ & $5 \mathrm{~mm}$ \\
\hline Number of slices & 9 (3 per plane) \\
\hline Slice gap & $1.5 \mathrm{~mm}$ \\
\hline Number of acquisitions $\left(N_{\mathrm{acq}}\right)$ & 1 \\
\hline Scan time & $23 \mathrm{sec}$ \\
\hline
\end{tabular}

A

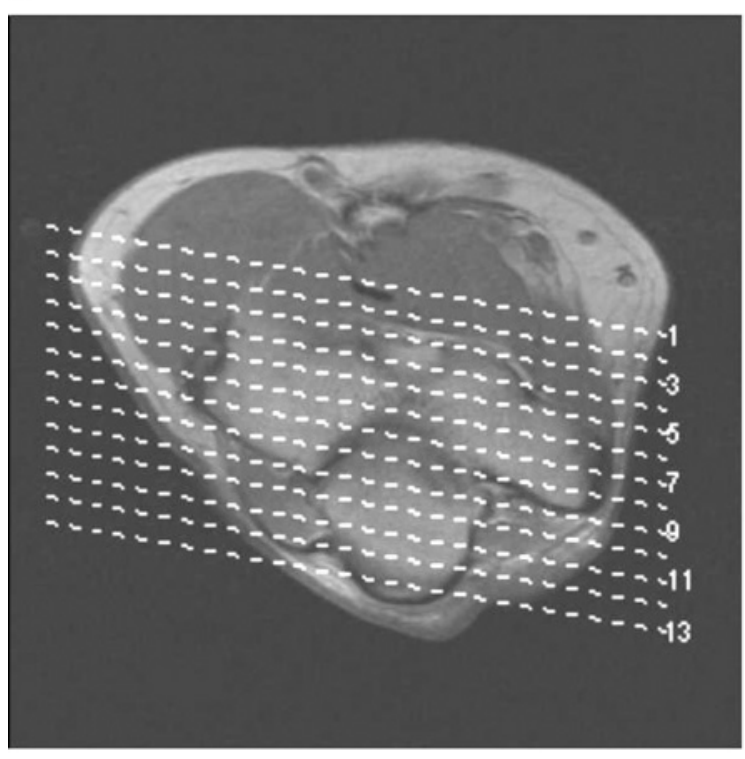

B

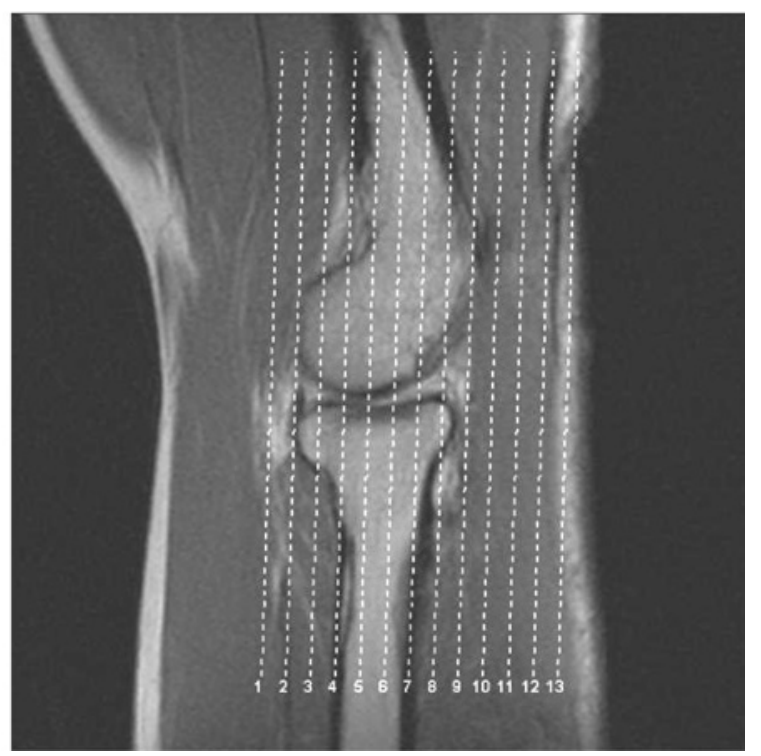

Figure A27.2.1 (A) Transverse scout for coronal images. Oblique coronal images are obtained parallel to a line connecting the humeral condyles anteriorly. (B) Sagittal scout for coronal images. Oblique coronal images are obtained parallel to a line along the long axis of the upper arm and forearm in the sagittal plane.

and perpendicular to the main field for a vertical-field magnet. Place sandbags on the arm and lateral to the arm in order to prevent potential motion of the arm.

A small-angle sponge may be placed under the handlforearm for very slight flexion of the elbow joint, which may allow for more comfort and therefore reduce potential motion.

8. Advance the scan table until the centering light is at the center of the RF coil. Evaluate for right/left and anterior/posterior offsets and use these values for localizer setup. Advance the patient to the isocenter of the magnet.

Elbow

A27.2.3

Supplement 14 
Table A27.2.3 Primary Clinical Imaging Parameters for Sequence 2 (Coronal $T_{1}$-Weighted)

\begin{tabular}{|c|c|}
\hline Patient position & Supine \\
\hline Scan type & Fast spin echo (FSE) \\
\hline Imaging plane (orientation) & Coronal oblique \\
\hline Central slice or volume center & Center of mass \\
\hline Echo time $\left(T_{\mathrm{E}}\right)$ & $17 \mathrm{msec}$ \\
\hline Echo train length (ETL) & 3 \\
\hline Repeat time $\left(T_{\mathrm{R}}\right)$ & $493 \mathrm{msec}$ \\
\hline Flip angle (FA) & $180^{\circ a}$ \\
\hline Fields of view $\left(\mathrm{FOV}_{\mathrm{x}}, \mathrm{FOV}_{\mathrm{y}}\right)$ & $110 \mathrm{~mm}, 110 \mathrm{~mm}$ \\
\hline Resolution $(\Delta x, \Delta y)$ & $0.43 \mathrm{~mm}, 0.54 \mathrm{~mm}$ \\
\hline Number of data points collected $\left(N_{\mathrm{x}}, N_{\mathrm{y}}\right)$ & $256,204^{b}$ \\
\hline Slice thickness $(\Delta z)$ & $3.5 \mathrm{~mm}$ \\
\hline Number of slices & 16 \\
\hline Slice gap & $0.4 \mathrm{~mm}$ \\
\hline Number of excitations (NEX) & $2^{c}$ \\
\hline Number of acquisitions $\left(\mathrm{N}_{\mathrm{acq}}\right)$ & 2 \\
\hline Scan time & $3 \mathrm{~min}, 24 \mathrm{sec}$ \\
\hline
\end{tabular}

Table A27.2.4 Primary Clinical Imaging Parameters for Sequence 3 (Coronal STIR)

\begin{tabular}{ll}
\hline Patient position & Supine \\
Scan type & Inversion recovery, fast spin echo \\
Imaging plane (orientation) & Coronal oblique \\
Central slice or volume center & Center of mass \\
Echo time $\left(T_{\mathrm{E}}\right)$ & $30 \mathrm{msec}$ \\
Echo train length (ETL) & 7 \\
Repeat time $\left(T_{\mathrm{R}}\right)$ & $4100 \mathrm{msec}$ \\
Inversion time $\left(T_{\mathrm{I}}\right)$ & $140 \mathrm{msec}$ \\
Flip angle $(\mathrm{FA})$ & $180^{\circ}$ \\
Fields of view $\left(\mathrm{FOV}_{\mathrm{x}}, \mathrm{FOV}_{\mathrm{y}}\right)$ & $110 \mathrm{~mm}, 110 \mathrm{~mm}$ \\
Resolution $(\Delta x, \Delta y)$ & $0.43 \mathrm{~mm}, 0.54 \mathrm{~mm}$ \\
Number of data points collected $\left(N_{\mathrm{x}}, N_{\mathrm{y}}\right)$ & $256,205^{a}$ \\
Slice thickness $(\Delta z)$ & $3.5 \mathrm{~mm}$ \\
Number of slices & 16 \\
Slice gap & $0.4 \mathrm{~mm}$ \\
Number of acquisitions $\left(N_{\mathrm{acq}}\right)$ & 1 \\
Scan time & $3 \mathrm{~min}, 45 \mathrm{sec}$ \\
\hline
\end{tabular}

${ }^{a}$ An additional $80 \%$ phase oversampling is applied.

Once this step has been performed, so long as the patient does not move on the table, the table itself can be moved and then returned to the same position as before without jeopardizing the positioning of one scan relative to another.

9. If the patient is unable to lie still, provide an appropriate sedative.

Masses of the Elbow and Forearm A27.2.4

\section{Sequence 1: Localizer}

10. Run the localizer (scout) to ensure the correct location of the elbow in three orientations using the imaging parameters in Table A27.2.2. 
A

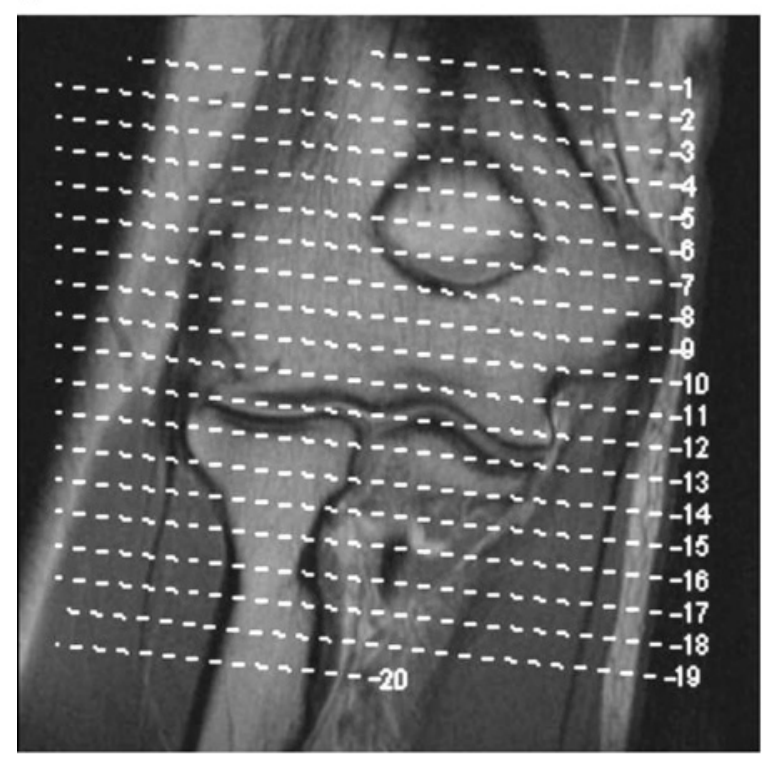

B

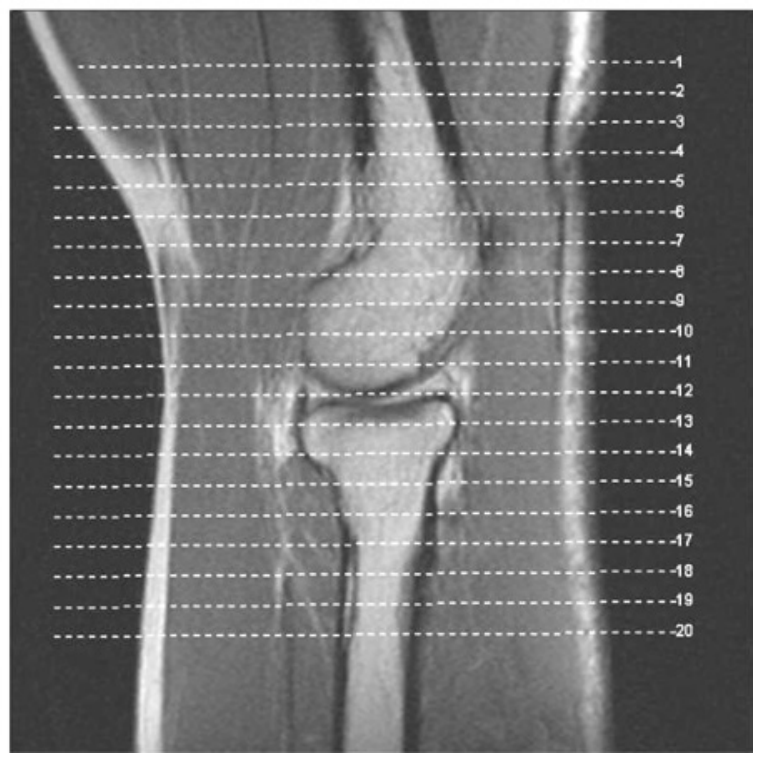

Figure A27.2.2 (A) Coronal scout for setting up transverse images. Transverse images should be obtained tangentially to the humeral condyle surface. Images may need to extend beyond routine parameters to include the entire mass lesion. (B) Sagittal scout for planning transverse images, which should be obtained orthogonally to the long axis of the elbow in the sagittal plane.

Table A27.2.5 Primary Clinical Imaging Parameters Sequence 4 (Transverse PDand $T_{2}$-Weighted)

\begin{tabular}{|c|c|}
\hline Patient Position & Supine \\
\hline Scan type & $\begin{array}{l}\text { Fast spin echo, dual echo (PD- and } \\
T_{2} \text {-weighted) }\end{array}$ \\
\hline Imaging plane (orientation) & Transverse \\
\hline Central slice or volume center & Center of mass \\
\hline Echo time $\left(T_{\mathrm{E}}\right)$ & $15 \mathrm{msec}$ and $92 \mathrm{msec}$ \\
\hline Echo train length (ETL) & 5 \\
\hline Repeat time $\left(T_{\mathrm{R}}\right)$ & $3000 \mathrm{msec}$ \\
\hline Flip angle (FA) & $150^{\circ a}$ \\
\hline Fields of view $\left(\mathrm{FOV}_{\mathrm{x}}, \mathrm{FOV}_{\mathrm{y}}\right)$ & $110 \mathrm{~mm}, 110 \mathrm{~mm}$ \\
\hline Resolution $(\Delta x, \Delta y)$ & $0.43 \mathrm{~mm}, 0.43 \mathrm{~mm}$ \\
\hline Number of data points collected $\left(N_{\mathrm{x}}, N_{\mathrm{y}}\right)$ & $256,256^{b}$ \\
\hline Slice thickness $(\Delta z)$ & $3 \mathrm{~mm}$ \\
\hline Number of slices & 16 \\
\hline Slice gap & $0.3 \mathrm{~mm}$ \\
\hline Number of acquisitions $\left(N_{\mathrm{acq}}\right)$ & 1 \\
\hline Scan time & $\sim 5 \min$ \\
\hline
\end{tabular}


A

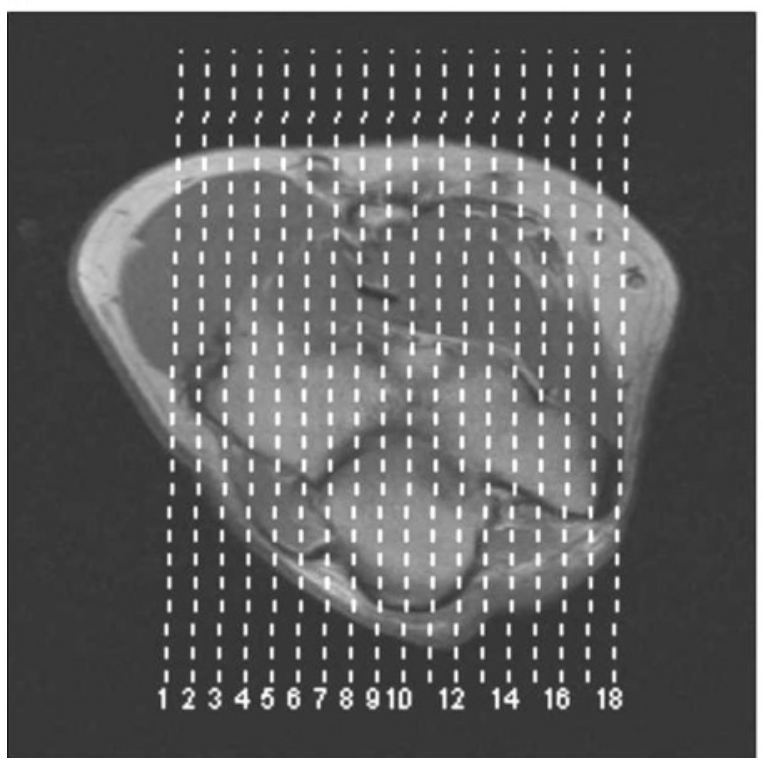

B

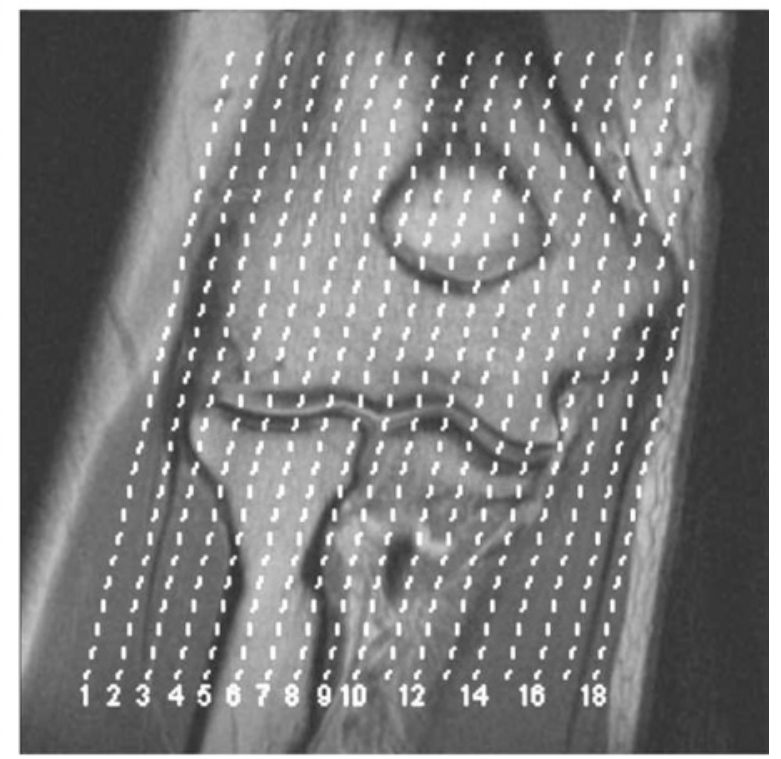

Figure A27.2.3 (A) Transverse scout for setting up sagittal images. Sagittal images are obtained perpendicular to coronal plane. The first and last images should include the epicondyle tips. (B) Coronal scout for planning sagittal images. Sagittal images should be parallel to the long axis of the elbow in the coronal plane of the scout image.

Table A27.2.6 Primary Clinical Imaging Parameters for Sequence 5 (Sagittal $T_{1}$-Weighted)

\begin{tabular}{ll}
\hline Patient position & Supine \\
Scan type & Fast spin echo \\
Imaging plane (orientation) & Sagittal \\
Central slice or volume center & Center of mass \\
Echo time $\left(T_{\mathrm{E}}\right)$ & $17 \mathrm{msec}$ \\
Echo train length (ETL) & 3 \\
Repeat time $\left(T_{\mathrm{R}}\right)$ & $493 \mathrm{msec}$ \\
Flip angle $(\mathrm{FA})$ & $180^{\circ a}$ \\
Fields of view $\left(\mathrm{FOV}_{\mathrm{x}}, \mathrm{FOV}_{\mathrm{y}}\right)$ & $110 \mathrm{~mm}, 110 \mathrm{~mm}$ \\
Resolution $(\Delta x, \Delta y)$ & $0.43 \mathrm{~mm}, 0.54 \mathrm{~mm}$ \\
Number of data points collected $\left(N_{\mathrm{x}}, N_{\mathrm{y}}\right)$ & $256,204^{b}$ \\
Slice thickness $(\Delta z)$ & $3.5 \mathrm{~mm}$ \\
Number of slices & 16 \\
Slice gap & $0.4 \mathrm{~mm}$ \\
Number of excitations $(\mathrm{NEX})$ & $2^{c}$ \\
Number of acquisitions $\left(N_{\mathrm{acq}}\right)$ & 2 \\
Scan time & $3 \mathrm{~min}, 24 \mathrm{sec}$ \\
\hline
\end{tabular}

${ }^{a}$ The system displays the flip angle of the refocusing pulse. The flip angle of the first pulse of this sequence is $90^{\circ}$.

${ }^{b}$ An additional $100 \%$ phase oversampling is applied.

${ }^{c}$ The number of concatenation is set to be 2 . This means that only half of the total slices will be excited during a given repeat time.

Masses of the 
Table A27.2.7 Primary Clinical Imaging Parameters for Sequence 6 (FatSuppressed $T_{2}$-Weighted)

\begin{tabular}{ll}
\hline Patient position & Supine \\
Scan type & Fast spin echo \\
Imaging plane (orientation) & Sagittal \\
Central slice or volume center & Center of mass \\
Echo time $\left(T_{\mathrm{E}}\right)$ & $44 \mathrm{msec}$ \\
Echo train length (ETL) & 5 \\
Repeat time $\left(T_{\mathrm{R}}\right)$ & $3000 \mathrm{msec}$ \\
Flip angle & $180^{\circ a}$ \\
Fields of view $\left(\mathrm{FOV}, \mathrm{V}_{\mathrm{x}}\right.$, FOV & $110 \mathrm{~mm}, 110 \mathrm{~mm}$ \\
Resolution $(\Delta x, \Delta y)$ & $0.43 \mathrm{~mm}, 0.43 \mathrm{~mm}$ \\
Number of data points collected $\left(N_{\mathrm{x}}, N_{\mathrm{y}}\right)$ & $256,256^{b}$ \\
Slice thickness $(\Delta z)$ & $3.5 \mathrm{~mm}$ \\
Number of slices & 20 \\
Slice gap & $0.4 \mathrm{~mm}$ \\
Number of acquisitions $\left(N_{\mathrm{acq}}\right)$ & 1 \\
Fat suppression & Yes \\
Scan time & $4 \mathrm{~min}, 53 \mathrm{sec}$ \\
\hline${ }^{2}$ The system displays the flip angle of the refocusing pulse. The flip angle of the first pulse of this sequence \\
is $90^{\circ}$. \\
${ }^{\circ}$ An additional $80 \%$ phase oversampling is applied.
\end{tabular}

\section{Sequence 2: Coronal $T_{1}$-weighted}

11. Use the localizer images to center at the joint line. Prescribe off transverse or sagittal scout images, in order to obtain coronal images (see Fig. A27.2.1). Run sequence 2 according to the parameters in Table A27.2.3.

Refer to the sample images in Figures A27.2.1A and B.

\section{Sequence 3: Coronal STIR}

12. Run sequence 3 according to the parameters in Table A27.2.4.

\section{Sequence 4: Transverse proton density weighted and $T_{2}$-weighted}

13. Prescribe off coronal or sagittal scout images in order to obtain transverse images (see Fig. A27.2.2).

14. Run sequence 4 according to the parameters in Table A27.2.5.

\section{Sequence 5: Sagittal $T_{1}$-weighted}

15. Prescribe off transverse or coronal scout images in order to obtain sagittal images (see Fig. A27.2.3).

16. Run sequence 5 according to the parameters in Table A27.2.6.

\section{Sequence 6: Sagittal fat-suppressed $T_{2}$-weighted}

17. Run sequence 6 according to the parameters in Table A27.2.7.

Also, an STIR sequence may be substituted for the sagittal fat saturated $T_{2}$-weighted sequence. An STIR sequence may be an alternative fat-suppression sequence, e.g., for mid/low-field scanners for which frequency-selective fat-saturation or fat suppression is not available, or for high-field scanners where field homogeneity is suspect and fat saturation is nonuniform. 


\section{ADDITIONAL FAT-SUPPRESSED SEQUENCES AND INTRAVENOUS POST-CONTRAST SEQUENCES}

Post-contrast images are optional and in many cases do not contribute to the diagnosis. However, intravenous gadolinium contrast agent may help to differentiate cystic or necrotic lesions from solid masses by defining a nodular or peripheral enhancing component in a cystic lesion versus more diffuse enhancement in a solid mass. In some cases, conversely, initial imaging without contrast agents may reveal the diagnosis and exclude the need for post-contrast imaging. Contrast agents may also be helpful in defining extent of a mass, but at times may be misleading for mass margins, as microscopic tumor involvement may extent beyond the macroscopic enhancing region of a mass. In general, the authors do not use contrast agents for evaluation of a mass lesion.

Standard, single-dose gadolinium contrast agent may be administered as an intravenous injection. Post-contrast $T_{1}$-weighted images (with fat suppression if available) can then be performed in transverse and coronal and/or sagittal planes. Pre-contrast $T_{1}$-weighted images should also be performed for comparison.

If a gadolinium i.v. contrast agent is to be used, the standard protocol is as follows: first, pre-contrast coronal or sagittal $T_{1}$-weighted images (depending on orientation of mass), and transverse proton-density- and $T_{2}$-weighted images, then, post-contrast coronal, sagittal, and transverse $T_{1}$-weighted images with fat suppression. The total scan time with contrast sequences is 30 to $45 \mathrm{~min}$, including injection time.

\section{Materials}

Normal saline $(0.9 \% \mathrm{NaCl})$, sterile

Gadolinium-based MR contrast agent (e.g., Magnevist, Omniscan, or Prohance)

21- to 23-G butterfly needle

\section{Set up patient and equipment}

1. Set up patient as in the Basic Protocol, steps 1 to 6.

2. Access a vein in the forearm not being imaged and, using a 21- to 23-G butterfly needle, establish an intravenous line through which the contrast agent can be injected.

It is preferable to insert the line prior to imaging and to leave the patient in the magnet, so that there is no intervening motion between the scans run before contrast agent injection and those run after injection.

3. Finish setting up the patient by performing steps 7 to 9 of the Basic Protocol.

4. Run sequences 1, 2 (or 5), and 4 in the Basic Protocol.

5. Without removing the patient from the magnet, inject the contrast agent and flush the intravenous line with $10 \mathrm{ml}$ saline solution.

A standard single dose of $0.1 \mathrm{mmol} / \mathrm{kg}$ of contrast agent is usually given.

\section{Sequence 7: Transverse fat-suppressed $T_{1}$-weighted}

6. Run sequence 7 according to the imaging parameters in Table A27.2.8.

\section{Sequence 8: Coronal fat-suppressed $T_{1}$-weighted}

7. Run sequence 8 according to the imaging parameters in Table A27.2.9.

\section{Sequence 9: Sagittal fat-suppressed $T_{1}$-weighted}

8. Run sequence 9 according to the imaging parameters in Table A27.2.9.

Masses of the 
Table A27.2.8 Primary Clinical Imaging Parameters for Sequence 7 (Transverse Fat-Suppressed $T_{1}$-Weighted)

\begin{tabular}{|c|c|}
\hline Patient position & Supine \\
\hline Scan type & Fast spin echo \\
\hline Imaging plane (orientation) & Transverse \\
\hline Central slice or volume center & Center of joint \\
\hline Echo time $\left(T_{\mathrm{E}}\right)$ & $19 \mathrm{msec}$ \\
\hline Echo train length (ETL) & 3 \\
\hline Repeat time $\left(T_{\mathrm{R}}\right)$ & $699 \mathrm{msec}$ \\
\hline Flip angle (FA) & $150^{\circ a}$ \\
\hline Fields of view $\left(\mathrm{FOV}_{\mathrm{x}}, \mathrm{FOV}_{\mathrm{y}}\right)$ & $110 \mathrm{~mm}, 110 \mathrm{~mm}$ \\
\hline Resolution $(\Delta x, \Delta y)$ & $0.43 \mathrm{~mm}, 0.43 \mathrm{~mm}$ \\
\hline Number of data points collected $\left(N_{\mathrm{x}}, N_{\mathrm{y}}\right)$ & $256,256^{b}$ \\
\hline Slice thickness $(\Delta z)$ & $3 \mathrm{~mm}$ \\
\hline Number of slices & 16 \\
\hline Slice gap & $0.3 \mathrm{~mm}$ \\
\hline Number of excitations (NEX) & $2^{c}$ \\
\hline Number of acquisitions $\left(N_{\mathrm{acq}}\right)$ & 1 \\
\hline Fat suppression & Yes \\
\hline Scan time & $4 \mathrm{~min}, 2 \mathrm{sec}$ \\
\hline
\end{tabular}

Table A27.2.9 Primary Clinical Imaging Parameters for Sequence 8 (Coronal and/or Sagittal Fat-Suppressed $T_{1}$-Weighted)

\begin{tabular}{ll}
\hline Patient position & Supine \\
Scan type & Fast spin echo \\
Imaging plane (orientation) & Coronal or sagittal \\
Central slice or volume center & Center of joint \\
Echo time $\left(T_{\mathrm{E}}\right)$ & $19 \mathrm{msec}$ \\
Echo train length (ETL) & 3 \\
Repeat time $\left(T_{\mathrm{R}}\right)$ & $699 \mathrm{msec}$ \\
Flip angle & $180^{\circ a}$ \\
Fields of view $\left(\mathrm{FOV}_{\mathrm{x}}, \mathrm{FOV}_{\mathrm{y}}\right)$ & $110 \mathrm{~mm}, 110 \mathrm{~mm}$ \\
Resolution $(\Delta x, \Delta y)$ & $0.43 \mathrm{~mm}, 0.43 \mathrm{~mm}$ \\
Number of data points collected $\left(N_{\mathrm{x}}, N_{\mathrm{y}}\right)$ & $256,256^{b}$ \\
Slice thickness $(\Delta z)$ & $3.5 \mathrm{~mm}$ \\
Number of slices & 16 \\
Slice gap & $0.4 \mathrm{~mm}$ \\
Number of excitations $(\mathrm{NEX})$ & $2^{c}$ \\
Number of acquisitions $\left(N_{\mathrm{acq}}\right)$ & 1 \\
Fat suppression & Yes \\
Scan time & $4 \mathrm{~min}, 2 \mathrm{sec}$ \\
\hline
\end{tabular}

${ }^{a}$ The system displays the flip angle of the refocusing pulse. The flip angle of the first pulse of this sequence is $90^{\circ}$.

${ }^{b}$ An additional $100 \%$ phase oversampling is applied.

${ }^{c}$ The number of concatenation is set to be 2 . This means that only half of the total slices will be excited during a given repeat time. 


\section{COMMENTARY}

\section{Background Information}

Musculoskeletal tumors of the elbow are unusual. By category, there are benign and malignant soft tissue and osseous masses. More commonly, benign masses occur as a result of acute trauma, chronic overuse, or arthropathy, and may simulate a tumor or more aggressive process. These include a retracted mass of torn tendon tissue, bursal collections, synovial or ganglion cysts, loose bodies, and scar tissue. Post-traumatic myositis ossificans presents as an area of soft-tissue swelling and/or hematoma that eventually undergoes a characteristic ossification pattern and may mimic a tumor. Synovial disorders (such as pigmented villonodular synovitis and synovial chondromatosis), gout, hemophilia, and neuropathic joints frequently present with masses about the joint. The most commonly occurring benign soft tissue tumors include lipomas, vascular tumors such as hemangiomas, and benign neural tumors, all of which often have definitive imaging characteristics and do not require postcontrast imaging. Malignant soft tissue tumors of the elbow are generally sarcomas and include liposarcoma, malignant fibrous histiocytoma, and synovial sarcoma. These tumors have nondefinitive imaging characteristics, but MR imaging may be vital in the evaluation of the extent of soft tissue involvement for tumor staging, as well as post-operative evaluation.

Benign and malignant osseous masses are generally better evaluated on plain film and computed tomography for specificity. However, MR imaging plays an important role in evaluation of soft tissue structures involved and for extent of bone/marrow involvement. The majority of bone tumors $(50 \%)$ occur in the ulna, with $20 \%$ occurring in the radius, $20 \%$ in the humerus, and $10 \%$ in the soft tissues. Overall, benign osseous tumors are more common. Benign tumors that can occur on the elbow, although unusual, include osteoblastomas, osteochondromas, chondroblastomas, and enchondromas. Additionally, fibrous dysplasia, giant cell tumors, aneurismal bone cysts, and unicameral bone cysts may occur. Subtle MR imaging findings of periostitis, nidus, and marrow edema may diagnose an unsuspected osteoid osteoma. The most common malignant osseous lesion about the elbow is metastasis. Plasmacytomas and multiple myeloma are the most common primary bone tumors, but much more commonly occur in the axial and proximal appendicular skeleton. Other primary malignant bone tumors include osteosarcoma, chondrosarcoma,
Ewing's sarcoma, and primary malignant lymphoma. MR imaging is most useful in delineating and staging these tumors (Ma, 1999; Steinbach et al., 1997a,b).

\section{Critical Parameters and Troubleshooting}

\section{Field of view}

Masses may extend beyond the normal fields of view (FOV) which may require the FOV to be increased to include all of the abnormality. Alternatively, additional imaging of the proximal humerus or distal forearm may provide the optimal evaluation without the sacrifice of resolution. In these instances, the elbow joint line should be included at the edge of the additional sagittal and/or coronal images to show the extent and location of the mass relative to the joint line.

\section{Contrast}

The need for post-contrast imaging is variable, as discussed in the Alternate Protocol.

\section{Anticipated Results}

The role of MRI in evaluating masses occurring on the elbow is to define the lesion by its location, intrinsic characteristics, and extent including structures involved. This information will lead the radiologist and the clinician to the diagnosis, differential diagnosis, or diagnostic category. It will also provide preoperative assessment or staging of the elbow when needed.

\section{Acknowledgement}

Special thanks to Phillip Becklund, Dr. Rajesh Sethi, and Susan Bybee for their time and contributions.

\section{Literature Cited}

Kneeland, J.B. 1997. MR Imaging of the elbow: Technical considerations. Magn. Reson. Imaging Clin. N. Am. 5:439-442.

Ma, L.D. 1999. Magnetic resonance imaging of musculoskeletal tumors: Skeletal and soft tissue masses. Curr. Probl. Diagn. Radiol. 28:29-62.

Shellock, F.G. 2001. MR Procedures: Health Effects and Safety. CRC Press, Boca Raton, Fla.

Steinbach, L.S., Fritz, R.C., Tirman, P.F., and Uffman, M. 1997a. Magnetic resonance imaging of the elbow. Eur. J. Radiol. 25:223-241.

Steinbach, L.S., Anderson, S., and Panicek, D. 1997b. MR imaging of musculoskeletal tumors in the elbow region. MRI Clin. N. Am. 5:619653.

Contributed by Jana Crain and Charles Ho National Orthopedic Imaging Associates

San Francisco, California 